\title{
Calculation of electron and hole impact ionization coefficients in SiGe alloys
}

\author{
K. Yeom, J. M. Hinckley, ${ }^{\text {a) }}$ and J. Singh \\ Department of Electrical Engineering and Computer Science, The University of Michigan, Ann Arbor, \\ Michigan 48109-2122
}

(Received 1 July 1996; accepted for publication 6 September 1996)

\begin{abstract}
Silicon-germanium alloys offer a system where the ratio of the electron impact ionization coefficient $(\alpha)$ and hole impact ionization coefficient $(\beta)$ varies from a value larger than unity (in high silicon content alloys), to a value smaller than unity (in high germanium content alloys). We report results for $\alpha$ and $\beta$ for this alloy system. The electron results are based on a multivalley nonparabolic band structure. The hole results are based on a six-band $k \cdot p$ model for low energies coupled to an eight-band model for high energies. We find that for the alloy $\mathrm{Si}_{0.4} \mathrm{Ge}_{0.6}, \alpha \sim \beta$. Alloy scattering is found to play an important role in determining the impact ionization coefficient. For compositions around $\mathrm{Si}_{0.5} \mathrm{Ge}_{0.5}$, the strong alloy scattering is found to suppress the impact ionization coefficient. (c) 1996 American Institute of Physics. [S0021-8979(96)03524-4]
\end{abstract}

\section{INTRODUCTION}

Over the last decade, remarkable progress has been made in the technology of SiGe epitaxy on a Si substrate. Many important challenges of dislocation control, $n$ - and $p$-type doping, Ohmic contacts, etc., have been resolved. The technology has matured to a stage where a number of groups have reported superb heterojunction bipolar transistor performances with cutoff frequencies approaching, or in some cases exceeding, $100 \mathrm{GHz} .{ }^{1}$ In addition to electronic devices, there is also considerable interest in avalanche photodiodes (APDs). High Ge content SiGe alloys can be used for detectors of long-haul communication optical signals (1.3 and $1.55 \mu \mathrm{m}$ wavelengths). Since these devices are compatible with Si technology, this could open doors for very low cost APDs.

In both electronic and optoelectronic devices, the impact ionization induced breakdown is of great importance. Electron-initiated impact ionization, $\alpha$, and hole-initiated impact ionization, $\beta$, limit the high power performance of electronic devices. Additionally, the ratio $\alpha / \beta$ is of great importance in the noise performance of APDs.

Although numerous device results have been reported on SiGe systems, there have been relatively few reports on the measurement and calculation of $\alpha$ and $\beta{ }^{2}$ In this paper, we report calculations of $\beta$ and $\alpha$ for SiGe alloys. In this study, we examine unstrained $\mathrm{SiGe}$ alloys. Such materials would be produced if the alloys were grown, for example, on a silicon substrate and the overlayer thickness was greater than the critical thickness. Our investigations focus on understanding the threshold energies for electron and hole impact ionization and on the calculation of $\alpha$ and $\beta$ values.

In the next section, we discuss the formalism used for our studies. In Sec. III, we present our results. Conclusions are given in Sec. IV.

\section{FORMALISM}

A general formalism for the calculation of impact ionization consists of the following components:

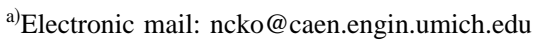

(1) An appropriate description for the band structure of the material. This is needed not only to describe the transport, but also to obtain the threshold energy for impact ionization.

(2) A description of the scattering processes, including a proper model for impact ionization.

(3) A description of carrier transport so that macroscopic quantities such as $\alpha$ and $\beta$ can be obtained.

In this section, we will describe the approach used for each of these.

\section{A. Band structure}

The band structure of a material is the most important component in a transport calculation. For the problem at hand, we use several different band-structure models, depending upon whether we are describing the conduction band or the valence band.

\section{Bandstructure for electron transport}

Silicon, germanium, and their alloys are all indirect band-gap materials. The bottom of the conduction band in $\mathrm{Si}$ occurs at $(0.85,0,0) 2 \pi / a$ (where $a$ is the lattice constant) and the five other equivalent points along the $\Gamma-\mathrm{X}$ direction. The six valleys produced are highly anisotropic. In Ge, the bottom of the conduction band occurs at the $L$ point and there are four equivalent conduction band valleys. For low electric field $(\leqslant 50 \mathrm{kV} / \mathrm{cm})$ in $\mathrm{Si}$, the inclusion of only the lowest valleys for electron transport is adequate. However, for the general alloy, and for high field transport (especially for breakdown studies), it is essential that both $L$ and $X$ valleys be included. Single analytical expressions for the $E-k$ relationship can be used for each of these valleys, since the band edges occur at different $k$ points and the valleys are not degenerate in $k$ space.

Near the bandedges, indirect gap semiconductors have the form,

$$
E(k)=E\left(k_{0}\right)+\frac{\hbar^{2}}{2}\left[\frac{\left(k_{l}-k_{l 0}\right)^{2}}{m_{l}^{*}}+\frac{\left(k_{t}-k_{t 0}\right)^{2}}{m_{t}^{*}}\right],
$$


TABLE I. Si and Ge conduction band parameters.

\begin{tabular}{clllclc}
\hline \hline Parameter & Symbol & Units & Si value & {$[$ Ref.] } & Ge value & {$[$ Ref.] } \\
\hline Nonparabolicity & $\alpha_{X}$ & $\mathrm{eV}^{-1}$ & 0.5 & 3 & 0.5 & $\ldots$ \\
& $\alpha_{L}$ & $\mathrm{eV}^{-1}$ & 0.3 & 4 & 0.33 & 4 \\
Effective mass & $m_{l X}^{*}$ & $m_{0}$ & 0.916 & 5 & 1.791 & 4 \\
& $m_{t X}^{*}$ & $m_{0}$ & 0.190 & 5 & 0.204 & 4 \\
& $m_{l L}^{*}$ & $m_{0}$ & 1.590 & 3 & 1.387 & 4 \\
\multirow{2}{*}{ Band edge } & $m_{t L}^{*}$ & $m_{0}$ & 0.126 & 4 & 0.101 & 4 \\
& $\epsilon_{X}$ & $\mathrm{eV}$ & 0 & $\ldots$ & 0.173 & 4 \\
& $\epsilon_{L}$ & $\mathrm{eV}$ & 1.05 & 4 & 0 & $\ldots$ \\
\hline \hline
\end{tabular}

where $m_{l}^{*}$ and $m_{t}^{*}$ are the longitudinal and transverse effective masses and $\left(k_{l 0}, k_{t 0}\right)$ represents the band edge $k$ point. Away from the band edges the bands are nonparabolic in nature. Therefore, it is convenient to use nonparabolic band structure to express the conduction band. The points $\left(k_{l 0}, k_{t 0}\right)$ are ignored in Monte Carlo simulation, because each conduction band valley is treated as an independent energy surface. Explicit tracking of the point $\left(k_{l 0}, k_{t 0}\right)$ is not needed in a Monte Carlo simulation since each conduction band valley is treated as an independent energy surface. Only insofar as the $f$ and $g$ scatterings are concerned does one need to keep track of the different valleys. An approximate description is given by

$$
\begin{aligned}
& \gamma(E)=E(1+\alpha E), \\
& =\frac{\hbar^{2}}{2}\left(\frac{k_{l}^{2}}{m_{l}^{*}}+\frac{k_{t}^{2}}{m_{t}^{*}}\right) .
\end{aligned}
$$

This expression is known to be valid for $L$ and $X$ conduction bands valleys. The values of the band parameters such as nonparabolicity $(\alpha)$, effective masses $\left(m_{l}^{*}\right.$ and $m_{t}^{*}$ for longitudinal and transverse effective mass, respectively), and valley separations between $X$ and $L$ valleys are given in Table $\mathrm{I}^{3-5}$

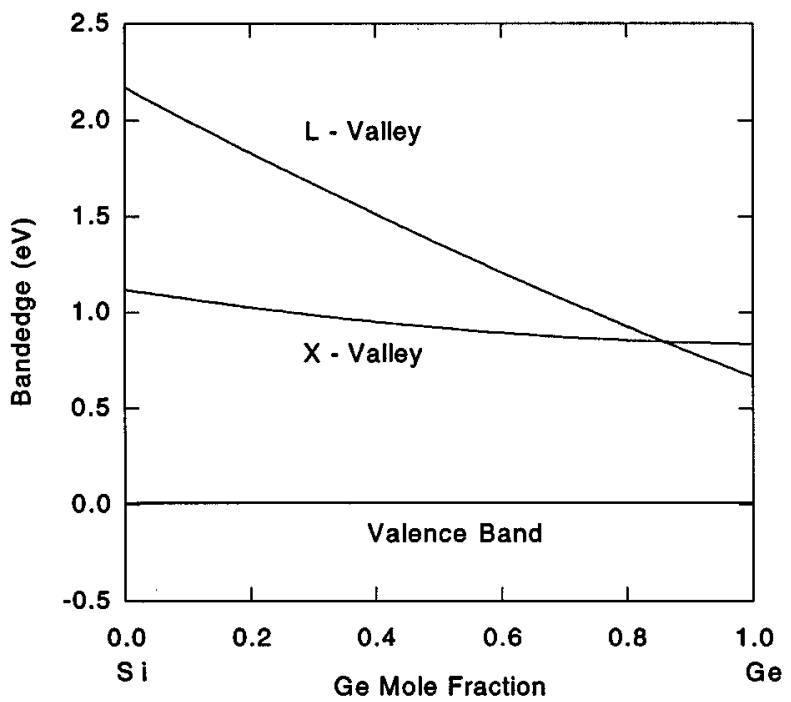

FIG. 1. Conduction band edge of the unstrained SiGe alloy, as a function of the germanium content.
TABLE II. Si and Ge valence band parameters.

\begin{tabular}{llccccc}
\hline \hline \multicolumn{1}{c}{ Parameter } & symbol & units & Si value & [Ref.] & Ge value & [Ref.] \\
\hline Spin-orbit splitting & $\Delta_{0}$ & $\mathrm{eV}$ & 0.044 & 10 & 0.282 & 11 \\
$k \cdot p$ parameters & $L$ & & -5.78 & 12 & -30.35 & 12 \\
& $M$ & & -3.44 & 12 & -4.85 & 12 \\
Deformation potential & $N$ & & -8.64 & 12 & -34.14 & 12 \\
& $a$ & $\mathrm{eV}$ & 2.1 & 13 & 2.0 & 13 \\
& $b$ & $\mathrm{eV}$ & -1.5 & 13 & -2.2 & 13 \\
& $d$ & $\mathrm{eV}$ & -3.4 & 13 & -4.4 & 13 \\
& $d_{0}$ & $\mathrm{eV}$ & 29.3 & 14 & 40.0 & 14 \\
\hline \hline
\end{tabular}

In the SiGe alloy we use the virtual crystal approximation to describe the band structure. According to this, the high symmetry points in the Brillouin zone are obtained from the $\mathrm{Si}$ and $\mathrm{Ge}$ values by the relation

$$
E_{\text {all }}\left(k_{i}\right)=(1-x) E_{\mathrm{Si}}\left(k_{i}\right)+x E_{\mathrm{Ge}}\left(k_{i}\right) .
$$

Although the conduction band structure is ellipsoid, it is mapped onto a sphere in the Monte Carlo simulation, through the Herring-Vogt transformation. ${ }^{3}$

The band parameters, such as the nonparabolicity and the effective mass, in $\mathrm{SiGe}$ are found by interpolation of those in $\mathrm{Si}$ and $\mathrm{Ge}$. To determine the energy values at the band edge, we use a quadratic relation with a bowing parameter. The bowing parameter is chosen to be 0.24 from experimental values. ${ }^{6}$ The energy separation from the valence band is given by

$$
\begin{aligned}
& \epsilon_{X}=1.12-0.523 x+0.24 x^{2}, \\
& \epsilon_{L}=2.17-1.746 x+0.24 x^{2},
\end{aligned}
$$

where $x$ is the Ge mole fraction. The band gap is chosen from the smaller of the two expressions given above. The $X$ and $L$ point band edges in the SiGe alloy are shown in Fig. 1. As can be seen, the alloy maintains $X$-like conduction band edge between 0 and $85 \% \mathrm{Ge}$. Beyond this, the bottom of the conduction band becomes $L$ like as in Ge.

TABLE III. Si and Ge TBM parameters.

\begin{tabular}{lllcc}
\hline \hline Parameter & Symbol & Units & Si value & Ge value \\
\hline TBM parameters & $V_{s 0}$ & $\mathrm{eV}$ & -4.19 & -5.8267 \\
from Ref. 21 & $V_{p 0}$ & $\mathrm{eV}$ & 0.2 & 0.6133 \\
& $V_{s 1}$ & $\mathrm{eV}$ & -4.19 & -5.8267 \\
& $V_{p 1}$ & $\mathrm{eV}$ & 0.2 & 0.6133 \\
& $V_{s 0 s 1 \sigma}$ & $\mathrm{eV}$ & -2.08 & -1.69 \\
& $V_{s 0 p 1 \sigma}$ & $\mathrm{eV}$ & 2.12 & 2.03 \\
& $V_{s 1 p 0 \sigma}$ & $\mathrm{eV}$ & 2.12 & 2.03 \\
& $V_{p 0 p 1 \sigma}$ & $\mathrm{eV}$ & 2.324 & 2.55 \\
& $V_{p 0 p 1 \pi}$ & $\mathrm{eV}$ & -0.517 & -0.67 \\
& $V_{s 0 s 0 \sigma}$ & $\mathrm{eV}$ & 0 & 0 \\
& $V_{s 0 p 0 \sigma}$ & $\mathrm{eV}$ & 0 & 0 \\
& $V_{p 0 p 0 \sigma}$ & $\mathrm{eV}$ & 0.58 & 0.41 \\
& $V_{p 0 p 0 \pi}$ & $\mathrm{eV}$ & -0.1 & -0.08 \\
& $V_{s 1 s 1 \sigma}$ & $\mathrm{eV}$ & 0 & 0 \\
& $V_{s 1 p 1 \sigma}$ & $\mathrm{eV}$ & 0 & 0 \\
& $V_{p 1 p 1 \sigma}$ & $\mathrm{eV}$ & 0.58 & 0.41 \\
& $V_{p 1 p 1 \pi}$ & $\mathrm{eV}$ & -0.1 & -0.08 \\
\hline \hline
\end{tabular}




\section{Bandstructure for hole transport: The $k \cdot p$ method}

To describe the hole transport it is not possible to use a simple analytical expression. This is due to the $k$-space degeneracy of the heavy-hole (HH) and light-hole (LH) states and their strong interaction. We use two approaches to describe the hole transport. For $k$ values close to the Brillouin zone center (up to $10 \%$ of the zone edge values) we use the $k \cdot p$ method. Beyond this, we use the tight-binding method (TBM). It is important to use the more accurate (i.e., with a better fit to the band-edge hole masses) $k \cdot p$ method at low fields. This ensures a good agreement with low field velocity-field results. Furthermore, even at high fields, a large portion of the carriers remain in the comparatively low-energy region of the band structure. By using this hybrid approach, the transport of the large body of carriers near the top of the band is treated accurately, while the impact ionization involving very high-energy carriers uses a band structure with reasonably accurate high-energy values.

In modeling the valence band structure for small $k$ values, we use a six-band $k \cdot p$ Hamiltonian with spin-orbit coupling. The band structure is obtained by calculating the eigenvalues of $H_{k p}+H_{s o}$. The $k \cdot p$ Hamiltonian matrix is: ${ }^{7}$

$$
\begin{aligned}
& H_{k p}=\left[\begin{array}{cc}
H^{\prime} & 0_{3 \times 3} \\
0_{3 \times 3} & H^{\prime}
\end{array}\right] \uparrow, \\
& H^{\prime}=-\frac{\hbar^{2}}{2 m_{0}}\left[\begin{array}{ccc}
L k_{x}^{2}+M\left(k_{y}^{2}+k_{z}^{2}\right) & N k_{x} k_{y} & N k_{z} k_{x} \\
N k_{x} k_{y} & L k_{y}^{2}+M\left(k_{z}^{2}+k_{x}^{2}\right) & N k_{y} k_{z} \\
N k_{z} k_{x} & N k_{y} k_{z} & L k_{z}^{2}+M\left(k_{x}^{2}+k_{y}^{2}\right)
\end{array}\right] \begin{array}{l}
x \\
y, \\
z
\end{array}
\end{aligned}
$$

where $m_{0}$ is the free electron mass. The dimensionless terms $L, M$, and $N$ are related to the Luttinger $\gamma$ parameters: ${ }^{8}$

$$
\begin{aligned}
& L=\gamma_{1}+4 \gamma_{2}, \\
& M=\gamma_{1}-2 \gamma_{2}, \\
& N=6 \gamma_{3} .
\end{aligned}
$$

The spin-orbit interaction is included by adding $H_{\text {so }}$ to $H_{k p}$. In the above basis, $H_{\text {so }}$ is ${ }^{9}$

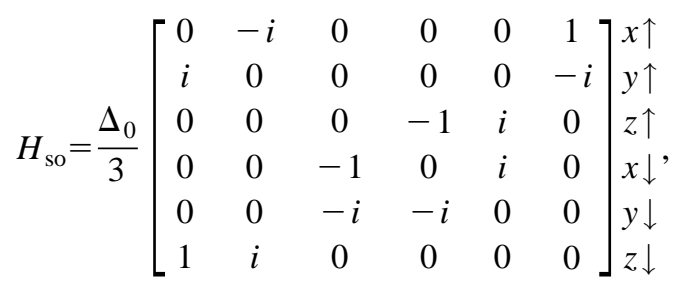

where $\Delta_{0}$ is the zone center $(k=0)$ spin-orbit splitting.

The effect of alloying is included through the virtual crystal approximation by a simple weighted average of the Kohn-Luttinger parameters. This band structure adequately describes the $\mathrm{HH}, \mathrm{LH}$, and split-off hole ( $\mathrm{SH})$ bands. In Table II, we give the important parameters to describe the valence band of $\mathrm{Si}$ and $\mathrm{Ge} .^{10-14}$

\section{Band structure for hole transport: Tight binding method}

The $k \cdot p$ method and its representation through the Kohn-Luttinger model discussed above gives a good description of the band structure for $k$ values up to $10 \%$ of the Brillouin zone edge values, which translates to energies of up to $\sim 0.5 \mathrm{eV}$. This implies that we can use the $k \cdot p$ method for transport up to fields of $\sim 50 \mathrm{kV} / \mathrm{cm}$, but not for fields approaching breakdown values. To study very high field transport, we have used the empirical tight-binding method for the band structure.
The empirical tight-binding method ${ }^{15}$ has proved useful in studying the electronic properties of solids. It has been applied to study the real and complex band structure of bulk materials, ${ }^{16,17}$ energy levels in superlattices, ${ }^{18,19}$ and electronic states and transport properties at interfaces. ${ }^{20}$ These properties are derived implicitly through matrix elements dependent on the tight-binding parameters of materials comprising the heterostructure. Choosing the latter to yield the correct band gaps and band curvatures at points of high symmetry in the bulk band structures of these materials ensures an accurate description of the electronic state in the heterostructure. This implicit treatment is particularly well suited to study electronic properties in the $\mathrm{SiGe}$ system, since the tight-binding parameters of SiGe for varying Ge content may be obtained from those of $\mathrm{Si}$ and Ge using the virtual crystal approximation.

In most semiconductors the $s p^{3}$ basis set is used for the expansion of the Wannier functions. An example is a tightbinding fit for $\mathrm{Si}$ and $\mathrm{Ge}$ using eight orbitals, four each for the two unit cell atoms, yielding $8 \times 8$ matrix representation for the bulk tight-binding Hamiltonian (without spin-orbit coupling). We employ this eight-band basis retaining up to second nearest-neighbor interactions. The tight-binding parameters used are given in Table III. ${ }^{21}$

In our transport formalism, we have used a combination of the $k \cdot p$ method for low-energy transport (to save computer time and maintain accuracy for low field transport) and the tight-binding method when the carrier energy is high. This hybrid model also includes a transition region in which the energy value in a band is a linear interpolation of the $k \cdot p$ and TBM values. An example band structure, for silicon, is shown in Fig. 2. In Fig. 2(a), the three regions are clearly evident. Figure 2(b) shows the differences, at higher energies, between the $k \cdot p$ and the tight-binding band structures. 



FIG. 2. (a) Schematic valence band, showing regions where $k \cdot p$ and tightbinding methods are used and the transition region between them. (b) Differences between the $k \cdot p$ (dashed lines) and tight-binding (solid lines) band structures.

\section{B. Scattering mechanisms}

The transport of carriers in semiconductor is dominated by a scattering mechanism. In fact, without scattering, the particle will simply undergo Bloch oscillations in the Brillouin zone. One can conceptually think of the particle transport as a series of free flights in the applied field followed by scatterings. The free flight trajectory of the electron is known through the equation

$$
\hbar \frac{d \mathbf{k}}{d t}=e \mathbf{F},
$$

where $\mathbf{F}$ is the electric field. The energy of the particle is simply obtained from the $E$ versus $k$ relation.

In Table IV, we show the various scattering processes that are of importance in $\mathrm{Si}, \mathrm{Ge}$, and $\mathrm{SiGe}$ alloy. Also mentioned are some of the important properties of the scattering mechanisms. In this section, we will briefly describe the scattering mechanism and present the expression for the scattering rate and its angular dependence. All of the scattering rates are based on the use of Fermi's Golden Rule for scattering. The scattering processes are similar for electrons and holes. For electrons, the overlap function of the basis states is unity and, therefore, need not be explicitly present in formulae for the scattering matrix elements. However, for holes, with coupling between six basis states, describing the wave functions of the three-coupled hole bands (heavy, light, and split-off), the overlap function is not unity and does depend on the initial and final wave vectors. The additional complexity arising from this coupling will be noted, where relevant, in the following discussion.
TABLE IV. Characteristics of primary electron and hole scattering processes in $\mathrm{Si}_{1-x} \mathrm{Ge}_{x}$.

\begin{tabular}{ll}
\hline \hline Scattering mechanism & \multicolumn{1}{c}{ Comments } \\
\hline Alloy scattering & $\begin{array}{l}\text { Arises from random potential fluctuation } \\
\text { in alloys. Is absent in Si and Ge. Alloy } \\
\text { potential is obtained from experiments. } \\
\text { Scattering is elastic. }\end{array}$ \\
Acoustic phonon scattering & $\begin{array}{l}\text { Arises from in-phase vibrations of the } \\
\text { two-unit cell atoms. For most cases, the } \\
\text { scattering is elastic. } \\
\text { Optical phonon scattering }\end{array}$ \\
Arises from out-of-phase vibrations of the \\
unit cell atoms. Scattering is inelastic. \\
This is the most important source of energy \\
loss. \\
Conized impurity scattering \\
Impact ionization
\end{tabular}

Two mechanisms of lattice scattering are used in this work: deformation potential acoustic phonon scattering and deformation potential (nonpolar) optical phonon scattering. Alloy scattering is included as an additional scattering mechanism in the alloy compositions studied. For holes, each scattering mechanism may drive both inter- and intraband transitions between and within the three valence bands. This results in three intraband and six interband modes of scattering for each mechanism.

Scattering rates for transitions from band $n$ (i.e., $\mathrm{HH}$, $\mathrm{LH}$, or $\mathrm{SH}$ ) to band $n^{\prime}$ (i.e., $\mathrm{HH}, \mathrm{LH}$, or $\mathrm{SH}$ ) by mechanism $m$ (i.e., acoustic phonon, optical phonon, or alloy scattering), are calculated using Fermi's second Golden Rule

$$
\begin{aligned}
W_{m ; n, n^{\prime}}(\mathbf{k})= & \frac{V_{c}}{(2 \pi)^{3}} \frac{2 \pi}{\hbar} \int d^{3} k^{\prime}\left|M_{m ; n, n^{\prime}}\left(\mathbf{k}, \mathbf{k}^{\prime}\right)\right|^{2} \\
& \times \delta\left(E_{n}+\Delta E_{m}-E_{n^{\prime}}^{\prime}\right),
\end{aligned}
$$

where $M$ is the scattering matrix element, $V_{c}$ is the crystal volume, and the density of final states is given by the delta function. Conservation of energy reduces this integral from three to two dimensions, with the integration being carried out over the constant energy surface $E=E_{n^{\prime}}^{\prime}$. Because of the complex warped nature of the valence band constant energy surfaces, the integration is done numerically. We have found a ten-point Newton-Cotes algorithm to work satisfactorily for the integration of each dimension separately.

As indicated by the form of the valence band Hamiltonian [Eqs. (7) and (8)], the valence band wave functions are composed of six basis states:

$$
|j\rangle \in\{|x \uparrow\rangle,|y \uparrow\rangle,|z \uparrow\rangle,|x \downarrow\rangle,|y \downarrow\rangle,|z \downarrow\rangle\} .
$$

A specific hole wave function, in band $n$, at wave vector $\mathbf{k}$, can be expressed as

$$
|n, \mathbf{k}\rangle=\sum_{j=1}^{6}|j\rangle\langle j \mid n, \mathbf{k}\rangle \text {. }
$$

The matrix elements of the scattering Hamiltonians are initially calculated in terms of the basis functions $|j\rangle$. Then, to 
obtain the scattering matrix elements for a specific transition from $|n, \mathbf{k}\rangle$ to $\left|n^{\prime}, \mathbf{k}^{\prime}\right\rangle$, the projection operators are used:

$$
M_{m ; n, n^{\prime}}\left(\mathbf{k}, \mathbf{k}^{\prime}\right)=\sum_{j, j^{\prime}=1}^{6}\left\langle n^{\prime}, \mathbf{k}^{\prime} \mid j^{\prime}\right\rangle\left\langle j^{\prime}\left|H_{m}\right| j\right\rangle\langle j \mid n, \mathbf{k}\rangle,
$$

where $H_{m}$ is the scattering Hamiltonian for mechanism $m$.

In previous publications, lattice scattering has been discussed in detail. Therefore, we will further elaborate only on alloy scattering and impact ionization scattering in this paper.

\section{Alloy scattering}

Alloy scattering is modeled after the work of Harrison and Hauser, ${ }^{22}$ treating the $\mathrm{Si}_{1-x} \mathrm{Ge}_{x}$ system as a random al- loy. The scattering potential, $\Delta U(\mathbf{r})$, is taken to be a spherically symmetric square well of depth $U_{\text {all }}$ and radius $r_{0}$, such that its spherical volume is $(4 \pi / 3) r_{0}^{3}=a_{0}^{3} / 4$. This represents the effect of band-structure fluctuations in the mixed alloy.

The alloy scattering rate for electrons in the conduction band can be represented in a simple form. The scattering matrix element is

$$
M\left(\mathbf{k}, \mathbf{k}^{\prime}\right)=\frac{\sqrt{N x(1-x)}}{V_{c}} \int e^{i\left(\mathbf{k}-\mathbf{k}^{\prime}\right) \cdot \mathbf{r}} \Delta U(\mathbf{r}) d \mathbf{r},
$$

where $N$ is the number of primitive cells in the crystal and $x$ is the germanium mole fraction. If $\mathbf{k}_{d}=\mathbf{k}^{\prime}-\mathbf{k}$ and $\theta$ is an angle between $\mathbf{k}$ and $\mathbf{k}^{\prime}$, then

$$
\begin{aligned}
M\left(\mathbf{k}, \mathbf{k}^{\prime}\right) & =\frac{\sqrt{N x(1-x)}}{V_{c}} \int e^{i k_{d} r \cos \theta} \Delta U(\mathbf{r}) d \mathbf{r} \\
& =U_{\text {all }} \frac{\sqrt{N x(1-x)}}{V_{c}} \int_{0}^{r_{0}} \int_{0}^{\pi} \int_{0}^{2 \pi} r^{2} e^{i k_{d} \cos \theta} \sin \theta d \phi d \theta d r=2 \pi U_{\text {all }} \frac{\sqrt{N x(1-x)}}{V_{c}} \int_{0}^{r_{0}} \int_{0}^{\pi} r^{2} e^{i k_{d} \cos \theta} \sin \theta d \theta d r \\
& =\frac{4 \pi U_{\text {all }}}{k_{d}^{2}} \frac{\sqrt{N x(1-x)}}{V_{c}} \int_{0}^{r_{0}} k_{d} r \sin \left(k_{d} r\right) d r=\frac{4 \pi r_{0}^{3}}{3} U_{\text {all }} \frac{\sqrt{N x(1-x)}}{V_{c}} \frac{3 \sin \left(k_{d} r_{0}\right)-3\left(k_{d} r_{0}\right) \cos \left(k_{d} r_{0}\right)}{\left(k_{d} r_{0}\right)^{3}} .
\end{aligned}
$$

For holes, the diagonal scattering matrix element between basis states $|j\rangle$ and $\left|j^{\prime}\right\rangle\left(j=j^{\prime}\right)$ is the same as the above expression for electrons. The off-diagonal terms are zero:

$$
\begin{aligned}
\left\langle j^{\prime}|H| j\right\rangle= & \delta_{j j^{\prime}} \frac{\sqrt{N x(1-x)}}{V_{c}} \\
& \times U_{\text {all }} \frac{3 \sin \left(k_{d} r_{0}\right)-3\left(k_{d} r_{0}\right) \cos \left(k_{d} r_{0}\right)}{\left(k_{d} r_{0}\right)^{3}} .
\end{aligned}
$$

When $k_{d} r_{0} \ll 1$, the matrix element is simplified to

$$
M\left(\mathbf{k}, \mathbf{k}^{\prime}\right) \approx \frac{4 \pi r_{0}^{3}}{3} U_{\text {all }} \frac{\sqrt{N x(1-x)}}{V_{c}} .
$$

Therefore, the matrix element is constant and independent of the magnitude of the difference wave vector, $k_{d}$, when the energy of carrier is low. The constant matrix element makes alloy scattering random, because it is not a function of $\theta$. On the other hand, at high energy or in alloy cluster, $k_{d} r_{0}$ cannot be ignored, since the complete form of the matrix element depends on the magnitude of $k_{d}$. Even if the carrier energy is high, the small $\theta$ leads to a small $k_{d}$ that means alloy scattering at high energy is a forward scattering. As the carrier energy decreases, the alloy scattering becomes random. Rule

The alloy scattering rate is given by Fermi's Golden

$$
W_{k k^{\prime}}=\frac{V_{c}}{8 \pi^{3}} \frac{2 \pi}{\hbar} \int\left|M\left(\mathbf{k}, \mathbf{k}^{\prime}\right)\right|^{2} \delta\left(\epsilon_{k}-\epsilon_{k^{\prime}}\right) d \mathbf{k}^{\prime},
$$

where $V_{c}$ is the crystal volume. If $\tau=k r_{0}$, then

$$
W_{k k^{\prime}}=\frac{16 \pi^{3} U_{0}^{2} r_{0}^{6}}{\hbar \tau^{2}} N\left(\epsilon_{k}\right) P(\tau)
$$

where $N\left(\epsilon_{k}\right)$ is a density of states and $P(\tau)$ is a function of wave vector (or energy) of initial state and converges to unity when $k r_{0}$ is small.

$$
\begin{aligned}
P(\tau)= & \frac{9}{(2 \tau)^{2}} \int_{0}^{2 \tau} \frac{(\sin t-t \cos t)^{2}}{t^{5}} d t \\
= & \frac{9}{8 \tau^{2}}\left(1-\frac{1}{(2 \tau)^{2}}+\frac{\sin 4 \tau}{(2 \tau)^{3}}-\frac{\sin ^{2} 2 \tau}{(2 \tau)^{4}}\right) \approx 1, \\
& \text { if } \tau \ll 1 .
\end{aligned}
$$

Therefore, the value of $P(\tau)$ stands for how much the alloy scattering deviates from the constant matrix element at low energy.

As a summary for carrier of low energy, (i) the alloy matrix element is constant, (ii) the matrix element is independent of the scattering angle, and the final state is randomly chosen. For carrier of high energy or in alloy cluster, (i) the alloy matrix element is $k_{d} r_{0}$ dependent, (ii) the matrix element is also a function of the scattering angle so that the alloy scattering becomes more forward scattering as the energy is increased, and (iii) the alloy scattering rate is smaller when it is compared with the value from the constant matrix element expression. 
TABLE V. Threshold energy for electron and hole impact ionization in pure silicon and pure germanium. Units are $\mathrm{eV}$ for all the values.

\begin{tabular}{lcc}
\hline \hline & Silicon & Germanium \\
\hline $\begin{array}{l}\text { Band-gap energy } \\
\text { Electron threshold energy } \\
X \text { valley }\end{array}$ & $1.120(1.1)$ & $0.664(0.7)$ \\
$\quad \begin{array}{l}L \text { valley } \\
\text { Hole threshold energy }\end{array}$ & $1.122(1.1)$ & $0.839(0.9)$ \\
HH & $\ldots$ & $0.823(0.8)$ \\
LH & $1.700(1.8)$ & $0.900(0.9)$ \\
SH & $1.703(2.1)$ & $0.994(1.0)$ \\
\hline \hline
\end{tabular}

\section{Impact ionization scattering}

Impact ionization starts to become an important scattering process once the carrier energies are high enough to cause interband transitions. This occurs at fields greater than $100 \mathrm{kV} / \mathrm{cm}$. The impact ionization is a carrier-carrier scattering process mediated by Coulombic scattering. While, in principle, this scattering is simple, in practice, there is no simple $a b$ initio derivation of the scattering rate that results in an analytical model. What can be derived is the threshold energy for the scattering process. The threshold energy arises from the energy and momentum conservation of the particles.

Focusing on the electron initiated breakdown, if $E_{c}\left(\mathbf{k}_{1}\right), E_{c}\left(\mathbf{k}_{2}\right), E_{v}\left(\mathbf{k}_{3}\right)$ are the energies (momentum) of the final electrons and hole and $E_{f}\left(\mathbf{K}_{f}\right)$ is the energy (momentum) of the initiating electron, we have

$$
E_{f}=E_{c}\left(\mathbf{k}_{1}\right)+E_{c}\left(\mathbf{k}_{2}\right)-E_{v}\left(\mathbf{k}_{3}\right),
$$

and

$$
\mathbf{K}_{f}=\mathbf{k}_{1}+\mathbf{k}_{2}-\mathbf{k}_{3},
$$

where $E_{c}(\mathbf{k})$ and $E_{v}(\mathbf{k})$ are energy of carriers at $\mathbf{k}$ in the conduction band and valence band, respectively, when electron impact ionization is considered. To minimize the total energy $E_{f}$ for a given momentum $\mathbf{K}_{f}$, the final carriers should have the same group velocity. ${ }^{23}$ With this condition and the given band structure, the threshold energy can be found.

Having established band structure, the threshold energy for impact ionization can be calculated. Table $\mathrm{V}$ represents the results for pure silicon and pure germanium with bandgap energy used in the calculation. The data in the parenthesis are the values from Ref. 24. For electron threshold energy, the threshold energy in silicon is almost the same as the band-gap energy. In addition, there is no threshold condition for electrons of the $L$ valley in silicon. On the other hand, germanium has a threshold condition in both $X$ and $L$ valleys and the threshold energy is slightly larger $(0.1-0.2 \mathrm{eV})$ than the band gap in the $L$ valley. For hole threshold energy, there are nine combinations of the band. The smallest threshold energy value in each band is listed in Table 5. In both Si and $\mathrm{Ge}$, the smallest threshold condition occurs in $\mathrm{HH}$.

Figure 3 shows the electron threshold energy results for the unstrained $\mathrm{Si}_{1-x} \mathrm{Ge}_{x}$ alloys. The solid lines are band-gap energies from the valence band edge to the $X$ and $L$ conduction band edges and the marks are the threshold energies in

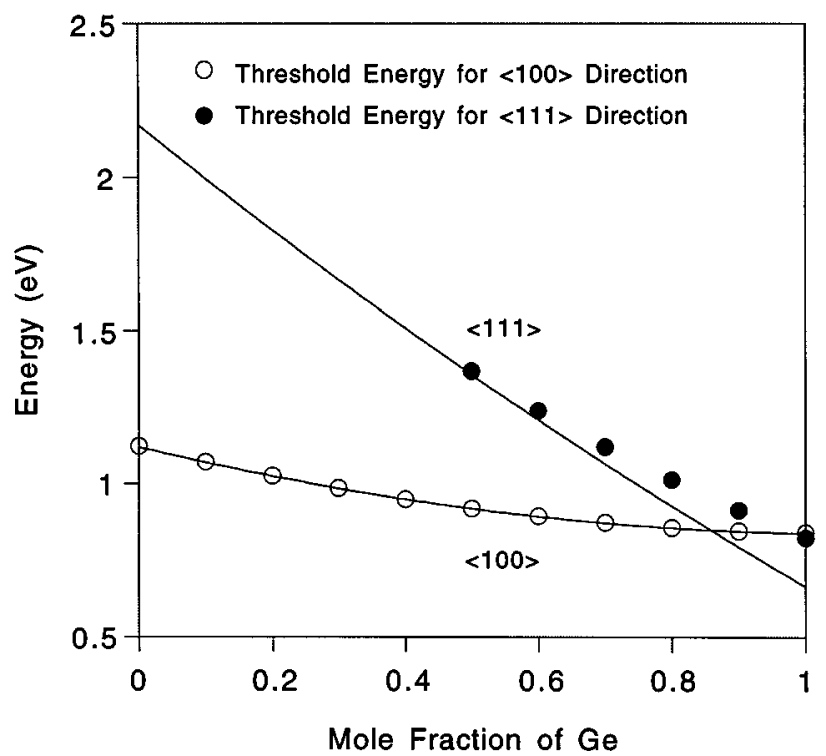

FIG. 3. Threshold energy for electron impact ionization in unstrained $\mathrm{Si}_{1-x} \mathrm{Ge}_{x}$ on a $\{100\}$ silicon substrate, shown as discrete points. Solid lines are the corresponding $X$-valley and $L$-valley band gaps.

each direction. For the $X$ valley in the unstrained case, the threshold energy exactly follows the band gap for the entire germanium mole fraction range considered. Therefore, the threshold energy in the $X$ valley can be simply expressed by Eq. (4). For electrons in the $L$ valley, the threshold energy is slightly larger than the band gap and there is no threshold condition from $0 \%$ to $50 \%$ germanium. The threshold energy in the $L$ valley is expressed by

$$
E_{\text {th } L}=0.48481 x^{2}-1.8159 x+2.1543, \quad \text { if } x \geqslant 0.5 \text {. }
$$

In the strained case, it is very important to note that the decrease of the heavy hole mass has no effect on the threshold energy because of the fact that final carriers lie approximately at the band edge where the group velocity is almost zero.

Figure 4 shows the hole threshold energy results for $\mathrm{Si}$, $\mathrm{Ge}$, and unstrained SiGe alloys. Alloys with a Ge mole fraction up to $50 \%$ and above $80 \%$, have the lowest threshold energy for heavy-hole-initiated impact ionization. In low Ge mole fraction alloys, an electron in the valence band interacts with a carrier in the $X$ valley of the conduction band, since it is the lowest type of conduction band valley and has the smallest energy separation with the valence band. As Ge mole fraction increases, the $L$-valley energy separation starts to decrease very sharply and becomes the dominant conduction valley for interaction of impact ionization. Because the threshold energy in $\mathrm{SH}$ is $0.4-0.5 \mathrm{eV}$ higher than that of the other bands, the heavy-hole and light-hole bands are the main sources of hole-initiated impact ionization.

As noted earlier, the total scattering rate cannot be determined $a b$ initio as a simple analytical model. However, several models, including the Keldysh model, the Thoma model, and the Cartier model, have been proposed to give a good description of the scattering rate.

The Keldysh model describes the impact ionization rate as 


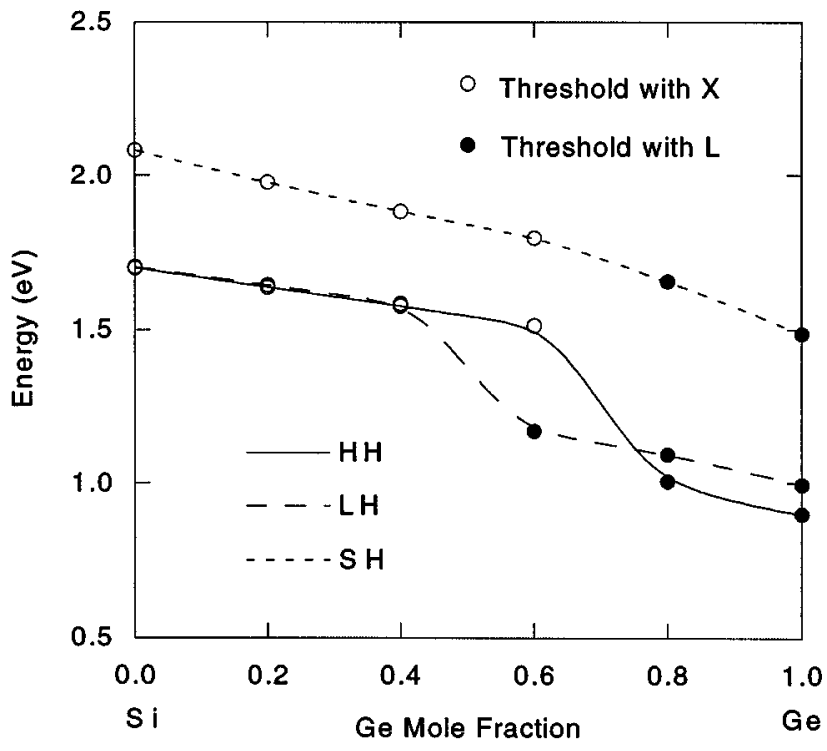

FIG. 4. Threshold energy for hole impact ionization in unstrained $\mathrm{Si}_{1-x} \mathrm{Ge}_{x}$.

$$
W_{\mathrm{imp}}(E)=\frac{P}{\tau\left(E_{\mathrm{th}}\right)}\left(\frac{E-E_{\mathrm{th}}}{E_{\mathrm{th}}}\right)^{2}
$$

where $P$ is the dimensionless parameter, $E_{\text {th }}$ is the threshold energy for impact ionization, and $1 / \tau\left(E_{\mathrm{th}}\right)$ is the phonon scattering rate averaged over all carrier wave vectors corresponding to the threshold energy $E_{\text {th }} . P$ is a fitting parameter that is found by comparison of the calculated value with the experimental data.

The Keldysh model has a $\left(E-E_{\text {th }}\right)^{2}$ energy dependence that arises from the integration over the final density of states in the scattering process. This is appropriate for electrons in direct band-gap materials. We use the Keldysh model for hole transport, but for electron transport, it is known that the initial breakdown is very "soft.", ${ }^{25}$ To represent the initial soft region, we use the Thoma model. ${ }^{26}$

The Thoma model introduces a combination of a set of expressions that represent the soft threshold near the threshold energy and harder threshold at high energy. It is expressed by

$$
W_{\text {imp }}(E)=\left\{\begin{array}{l}
0 \quad \text { if } E<E_{\text {th } 1} \\
W_{2}(E) \quad \text { if } W_{2}(E) \geqslant W_{3}(E), \\
W_{3}(E) \quad \text { if } W_{2}(E)<W_{3}(E)
\end{array}\right.
$$

where
TABLE VII. Si and Ge $X-X$ electron intervalley scattering parameters.

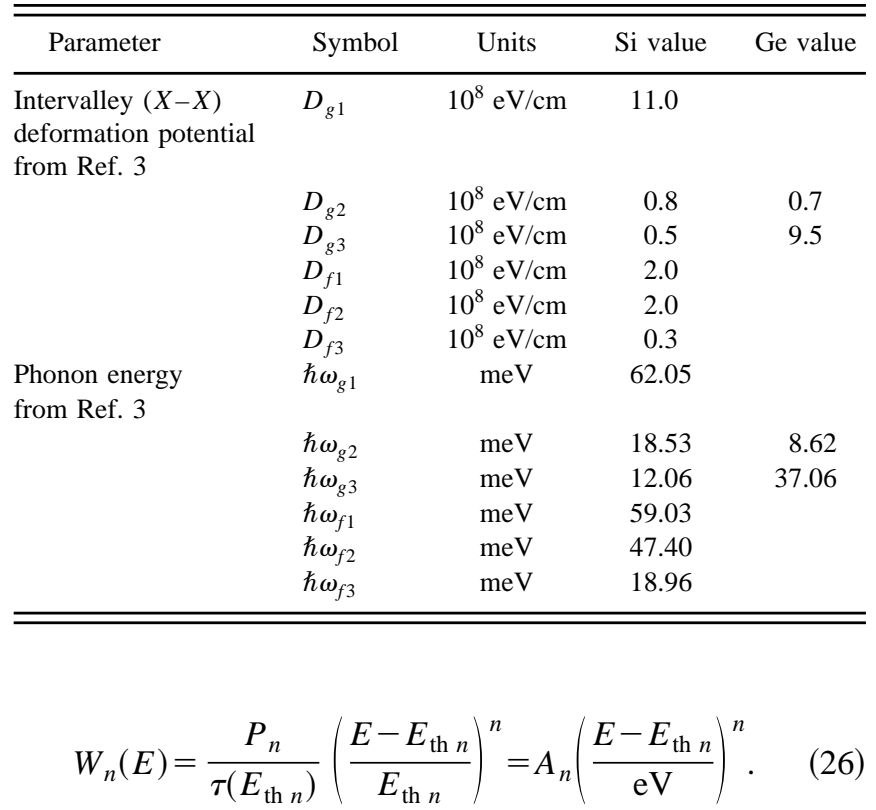

Here, we have grouped all of the factors that do not depend on the carrier's energy $E$, into the term $A_{n}$. The cubic relation around the threshold energy describes the "super" soft region near the threshold. The parameter $P$, or equivalently $A_{n}$, was rescaled for the band structure and scattering rates used in this work because of the overestimated value in the Thoma model. This was done by adjusting the values of $A_{n}$ to achieve a good agreement between the simulation results for silicon and the measured results from the literature. Specifically, the values used for $A_{n}$ were: $A_{2}=8.78 \times 10^{12} \mathrm{~s}^{-1}$ and $A_{3}=5.58 \times 10^{12} \mathrm{~s}^{-1}$.

\section{Transport formalism}

The formalism used to study the carrier transport is based on the Monte Carlo method. The electron Monte Carlo program is relatively simple and is based on the methods outlined in Refs. 3 and 27. The final state wave vector after impact ionization is chosen from a random distribution of states with average energy equal to $3 k_{B} T / 2$. The final states after scattering by all of the other processes (phonon and alloy scattering), are determined in the customary manner, using the differential scattering rate of the scattering mechanism involved. In Tables VI, VII, VIII, we give all of the important parameters used in the electron Monte Carlo simulation.

\begin{tabular}{|c|c|c|c|c|c|c|}
\hline Parameter & Symbol & Units & Si value & [Ref.] & Ge value & [Ref.] \\
\hline \multirow{2}{*}{$\begin{array}{l}\text { Acoustic phonon } \\
\text { deformation potential }\end{array}$} & $D_{X}$ & $\mathrm{eV}$ & 9.9 & $\cdots$ & 9.9 & $\cdots$ \\
\hline & $D_{L}$ & $\mathrm{eV}$ & 8.5 & $\cdots$ & 8.5 & $\cdots$ \\
\hline Phonon speed & $S$ & $10^{5} \mathrm{~cm} / \mathrm{s}$ & 9.04 & 5 & 5.0 & $\cdots$ \\
\hline \multirow{2}{*}{$\begin{array}{l}\text { Nonpolar optical phonon } \\
\text { deformation potential }\end{array}$} & $D_{0 X}$ & $10^{8} \mathrm{eV} / \mathrm{cm}$ & 4.51 & $\ldots$ & 4.51 & $\cdots$ \\
\hline & $D_{0 L}$ & $10^{8} \mathrm{eV} / \mathrm{cm}$ & 6.80 & $\cdots$ & 6.80 & $\cdots$ \\
\hline Phonon energy & $\hbar \omega_{0}$ & $\mathrm{meV}$ & 63.34 & 3 & 37.06 & 3 \\
\hline
\end{tabular}

TABLE VI. Si and Ge electron phonon scattering parameters. 
TABLE VIII. Si and Ge $X-L, L-L$ electron intervalley scattering parameters.

\begin{tabular}{|c|c|c|c|c|c|c|}
\hline Parameter & Symbol & Units & Si value & [Ref.] & Ge value & [Ref.] \\
\hline \multirow{4}{*}{$\begin{array}{l}\text { Intervalley }(X-L) \\
\text { deformation potential }\end{array}$} & $D_{X L 1}$ & $10^{8} \mathrm{eV} / \mathrm{cm}$ & 2.0 & 5 & 4.1 & 3 \\
\hline & $D_{X L 2}$ & $10^{8} \mathrm{eV} / \mathrm{cm}$ & 2.0 & 5 & & \\
\hline & $D_{X L 3}$ & $10^{8} \mathrm{eV} / \mathrm{cm}$ & 2.0 & 5 & & \\
\hline & $D_{X L 4}$ & $10^{8} \mathrm{eV} / \mathrm{cm}$ & 2.0 & 5 & & \\
\hline \multirow[t]{4}{*}{ Phonon energy } & $\hbar \omega_{X L 1}$ & $\mathrm{meV}$ & 57.91 & 5 & 27.58 & 3 \\
\hline & $\hbar \omega_{X L 2}$ & $\mathrm{meV}$ & 54.64 & 5 & & \\
\hline & $\hbar \omega_{X L 3}$ & $\mathrm{meV}$ & 41.36 & 5 & & \\
\hline & $\hbar \omega_{X L 4}$ & $\mathrm{meV}$ & 16.98 & 5 & & \\
\hline \multirow{2}{*}{$\begin{array}{l}\text { Intervalley }(L-L) \\
\text { deformation potential }\end{array}$} & $D_{L L 1}$ & $10^{8} \mathrm{eV} / \mathrm{cm}$ & 3.0 & $\cdots$ & 3.0 & 3 \\
\hline & $D_{L L 2}$ & $10^{8} \mathrm{eV} / \mathrm{cm}$ & 0.2 & $\cdots$ & 0.2 & 3 \\
\hline \multirow[t]{2}{*}{ Phonon energy } & $\hbar \omega_{L L 1}$ & $\mathrm{meV}$ & 27.58 & $\cdots$ & 27.58 & 3 \\
\hline & $\hbar \omega_{L L 2}$ & $\mathrm{meV}$ & 10.34 & $\cdots$ & 10.34 & 3 \\
\hline
\end{tabular}

The hole Monte Carlo is somewhat more complex, due to the highly anisotropic nature of the valence band structure. The approach used is based on the techniques developed by Hinckley and Singh. ${ }^{28-31}$ These references also contain the values of the material parameters used. The approach has been extended to include the eight-band tight-binding model for the band structure when the hole energies are above $0.5 \mathrm{eV}$.

\section{RESULTS}

In this section we will discuss the results for $\alpha$ and $\beta$. As noted in the introduction, our focus will be on high Ge content $\mathrm{SiGe}$ alloys.

\section{A. Impact ionization coefficients in $\mathrm{Si}$ and $\mathrm{Ge}$}

Before beginning the calculation of the impact ionization coefficient in the $\mathrm{Si}_{1-x} \mathrm{Ge}_{x}$ alloy, Monte Carlo simulations were performed for pure silicon to confirm the scattering parameters. Because the electron impact ionization rate near the threshold in indirect material is very soft and has a thirdorder dependence upon $\left(E-E_{\text {th }}\right)$ (Ref. 25) rather than a second-order dependence like the Keldysh model, ${ }^{32}$ the Thoma model ${ }^{26}$ is used in the calculation to describe the "super" soft region near the threshold. In Fig. 5, the Monte Carlo results for electrons in pure $\mathrm{Si}$ are shown. Also shown are experimental results. ${ }^{24,33,34}$ The calculated values show a good agreement with the experimental data from (Ref. 35), which is known to be one of the most refined sets of measurements. For high $\mathrm{Si}$ content $\mathrm{SiGe}$ alloys we will only focus on $\alpha$ since $\beta$ is almost an order of magnitude smaller.

Figure 6 shows the calculated impact ionization coefficient of electrons and holes in Ge. We show two sets of experimental data. ${ }^{36,37}$ There is some disparity between the published experimental results, although both results show a similar ratio between $\beta$ and $\alpha$ with $\beta$ always larger than $\alpha$. As can be seen from Fig. 6, our results fall within the experimental results.

\section{B. Impact ionization coefficients in SiGe alloys}

Having validated our Monte Carlo model, we now consider the $\mathrm{Si}_{1-x} \mathrm{Ge}_{x}$ alloy. As noted earlier, we will consider the case where the alloy is unstrained. This situation would arise if a thick (several microns) layer of $\mathrm{Si}_{1-x} \mathrm{Ge}_{x}$ was grown on a silicon substrate. We assume that effects of any dislocations that may be present in the alloy are negligible.

Monte Carlo parameters with the calculated threshold energy are used to study the impact ionization in $\mathrm{Si}_{1-x} \mathrm{Ge}_{x}$ alloy. In the $\mathrm{Si}_{1-x} \mathrm{Ge}_{x}$ alloy, an important additional scattering is the alloy scattering. Recently, the alloy scattering has been described by a set of parameters $U_{\text {all }}$ and $r_{0}$, where assuming a perfectly random alloy, it has been found that $U_{\text {all }}=0.7 \mathrm{eV}$ and $r_{0}=\sqrt{3} a / 4(2.36 \AA) .{ }^{27}$ To show the importance of alloy scattering, in Fig. 7, we give results for impact ionization in $\mathrm{Si}_{0.9} \mathrm{Ge}_{0.1}$ with and without alloy scattering. We see that when alloy scattering is included, the impact ionization is suppressed by about $20 \%$. Also, the effect of alloy scattering is more important at lower fields.

Before discussing the results for $\alpha$ and $\beta$, it is useful to examine, on a physical basis, what is to be expected. As the Ge content is increased, the band gap decreases. On this

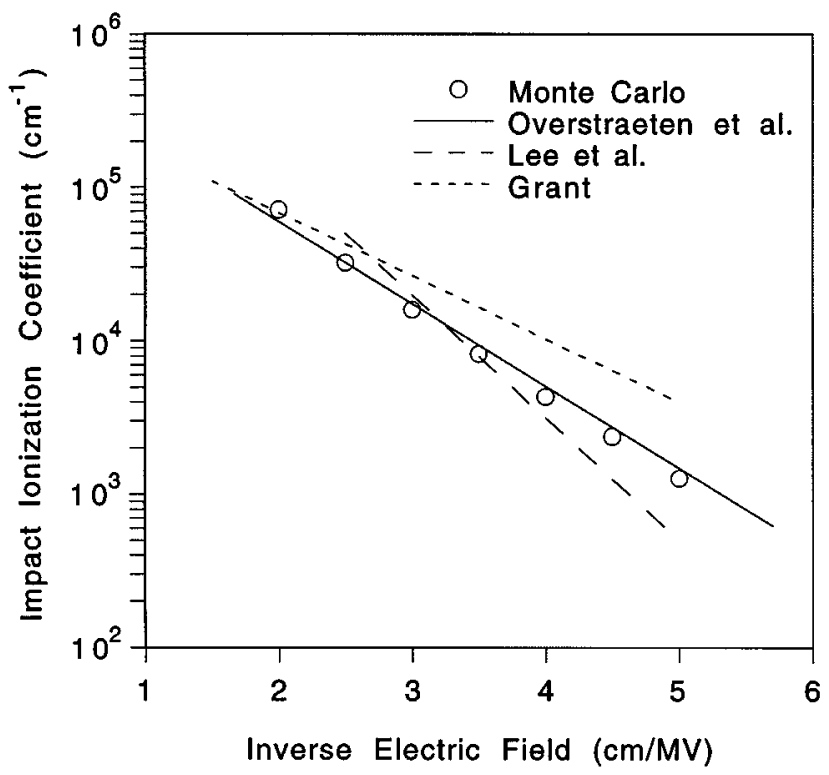

FIG. 5. Electron impact ionization coefficient from Monte Carlo simulations, compared with experimental data in pure silicon. 


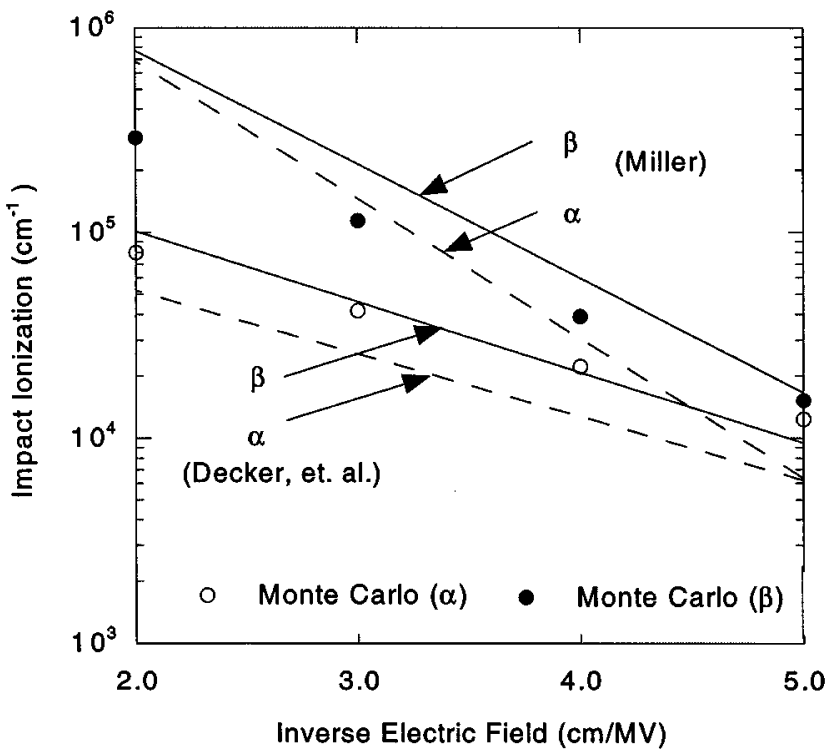

FIG. 6. Electron and hole impact ionization coefficient from Monte Carlo simulations, compared with experimental data in pure germanium.

basis, we expect that the impact ionization should increase for a given field. However, as the Ge composition is increased, the alloy scattering increases up to a Ge content of $50 \%$. The increasing alloy scattering randomizes the carrier distribution and suppresses the energy that the carriers can gain from the field. This, in turn, tends to suppress the impact ionization. Thus, there is a competition between the effect of decreasing band gap and increasing alloy scattering. However, once the Ge content exceeds $50 \%$, the effect of alloy scattering weakens.

In Fig. 8, we show the impact ionization for electrons in SiGe as a function of alloy composition at electric field values of 333 and $500 \mathrm{kV} / \mathrm{cm}$. We note that as the Ge content is increased from zero, initially the values of $\alpha$ remain almost unchanged, even though the band gap is shrinking. At Ge

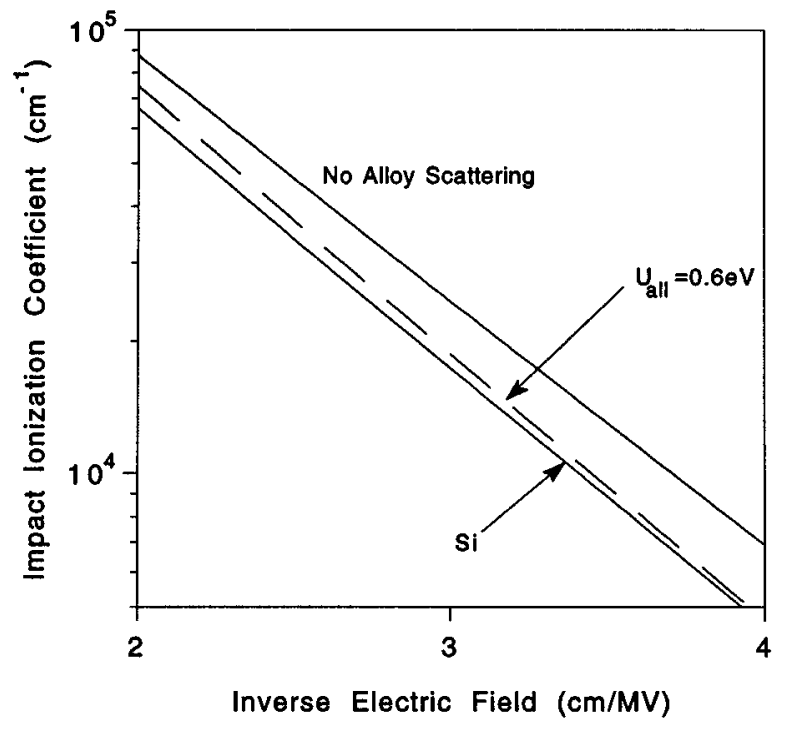

FIG. 7. Electron impact ionization coefficients for $\mathrm{Si}_{0.9} \mathrm{Ge}_{0.1}$ with and without alloy scattering. The results are compared with results for silicon.

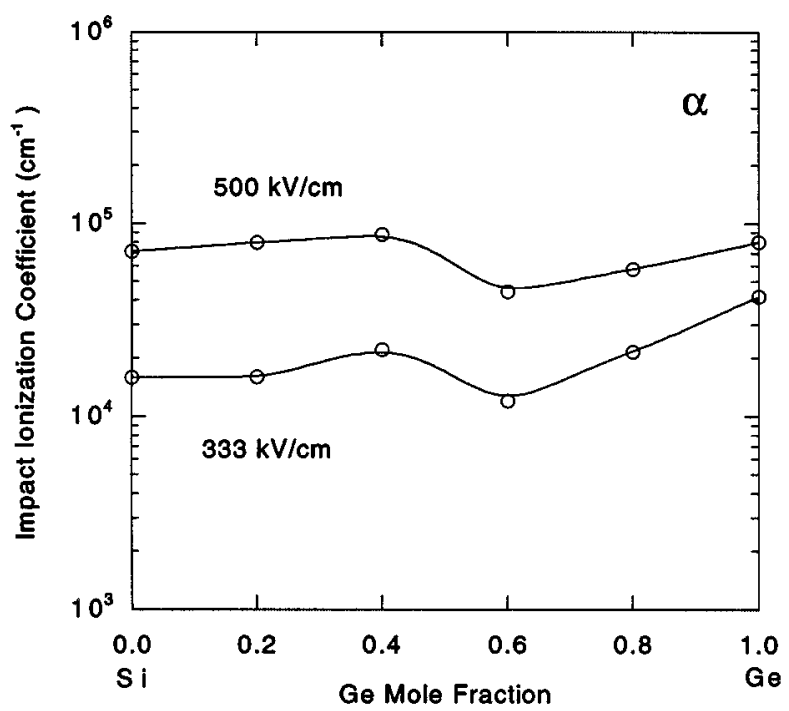

FIG. 8. The effect of Ge mole fraction on the electron and hole impact ionization coefficient.

compositions between 0.4 and 0.6 , the impact ionization decreases somewhat before again increasing. The reasons, as discussed above, have to do with the alloy scattering.

In Fig. 9, we show the hole impact ionization for Ge-rich alloys. There is a steady increase in $\beta$ values as the Ge content is increased. This is primarily the result of the sharply decreasing threshold energy for impact ionization as shown in Fig. 4.

Finally, in Fig. 10, we show the $\beta / \alpha$ ratio for the alloys as a function of composition. Also shown are some experimental results (along with error bars) from published data. ${ }^{2}$ The $\beta / \alpha$ ratio approximately equals 3 in Ge and we see that it steadily decreases as the Ge content in the alloy is decreased. It reaches a value of approximately 0.8 for $\mathrm{Si}_{0.4} \mathrm{Ge}_{0.6}$.

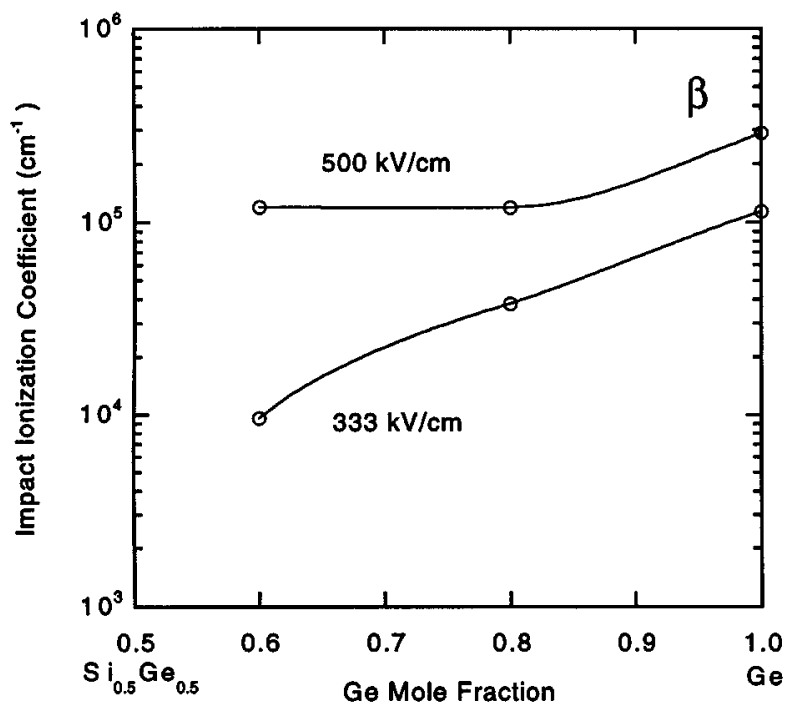

FIG. 9. The effect of Ge mole fraction on the electron and hole impact ionization coefficient. 


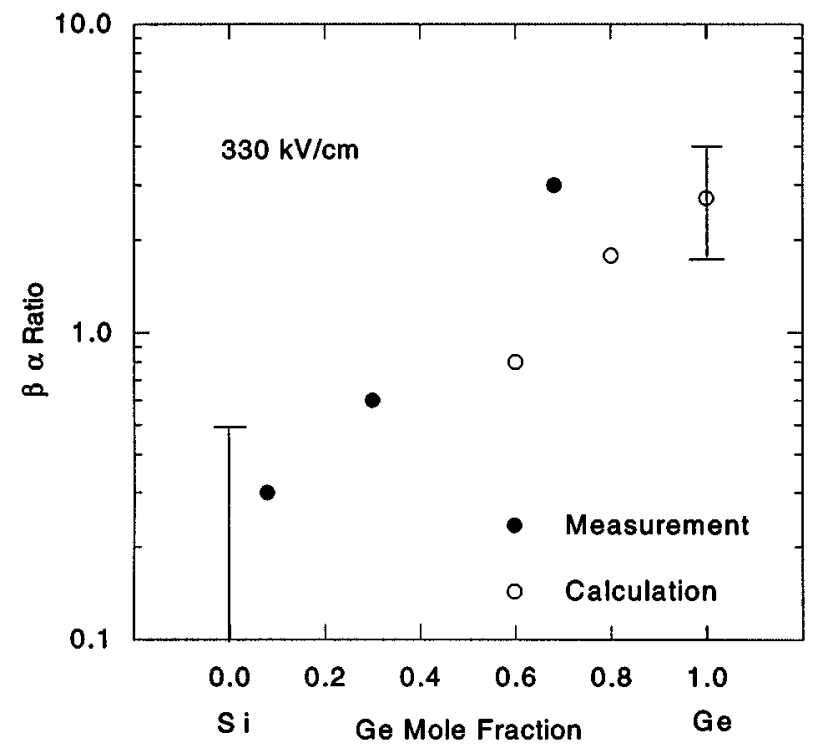

FIG. 10. The $\beta / \alpha$ ratio with Ge mole fraction at $330 \mathrm{kV} / \mathrm{cm}$. The Monte Carlo simulation results are compared with the experimental data from Ref. 2.

\section{CONCLUSIONS}

In this paper, we have calculated the electron and hole impact ionization coefficients in SiGe alloys. The importance of alloy scattering in suppressing impact ionization has been identified. Due to the alloy scattering, $\alpha$ does not increase from its value in $\mathrm{Si}$, as the $\mathrm{Ge}$ mole fraction is increased. Hole impact ionization has been calculated for $\mathrm{Si}_{1-x} \mathrm{Ge}_{x}$ with $x \geqslant 0.6$. We find that for $\mathrm{Si}_{0.4} \mathrm{Ge}_{0.6}$, the $\beta / \alpha$ ratio is approximately equal to 0.8 and it reaches a value of approximately 3 for Ge. Our results show good agreement with published measured results on the $\beta / \alpha$ ratios in $\mathrm{Si}$ and in $\mathrm{Ge}$.

\section{ACKNOWLEDGMENT}

This work was funded by the U.S. Army URI program (DAAL03-92-G-0109) by the U.S. Air Force (Grant No. AFOSR-91-0349).
${ }^{1}$ T. C. Chen, K. Y. Toh, J. D. Cressler, J. Warnock, P. F. Lu, D. D. Tang, G. P. Li, C. T. Chuang, and T. H. Ning, IEEE Electron Device Lett. 10, 364 (1989).

${ }^{2}$ J. Lee, A. L. Gutierrez-Aitken, S. H. Li, and P. K. Bhattacharya, Appl. Phys. Lett. 66, 204 (1995).

${ }^{3}$ C. Jacoboni and L. Reggiani, Rev. Mod. Phys. 55, 645 (1983).

${ }^{4}$ M. V. Fischetti, IEEE Trans. Electron Devices 38, 634 (1991).

${ }^{5}$ K. Hess, Advanced Theory of Semiconductor Devices (Prentice-Hall, Englewood Cliffs, NJ, Hall, 1988).

${ }^{6}$ J. S. Kline, F. H. Pollak, and M. Cardona, Helv. Phys. Acta 41, 968 (1968).

${ }^{7}$ G. Dresselhaus, A. F. Kip, and C. Kittel, Phys. Rev. 98, 368 (1955).

${ }^{8}$ J. M. Luttinger, Phys. Rev. 102, 1030 (1956).

${ }^{9}$ M. Tiersten, IBM J. Res. Dev. 5, 122 (1961).

${ }^{10}$ S. Zwerdling, K. J. Button, B. Lax, and L. M. Roth, Phys. Rev. Lett. 4, 173 (1960).

${ }^{11}$ S. H. Groves, C. R. Pidgeon, and J. Feinleib, Phys. Rev. Lett. 17, 643 (1966).

${ }^{12}$ P. Lawaetz, Phys. Rev. B 4, 3460 (1971).

${ }^{13}$ J. D. Wiley, Solid State Commun. 8, 1865 (1970).

${ }^{14}$ F. Szmulowicz, Phys. Rev. B 28, 5943 (1983).

${ }^{15}$ J. C. Slater and G. F. Koster, Phys. Rev. 94, 1498 (1954).

${ }^{16}$ D. J. Chadi and M. L. Cohen, Phys. Status Solidi B 68, 405 (1975).

${ }^{17}$ Y.-C. Chang, Phys. Rev. B 25, 605 (1982).

${ }^{18}$ J. N. Schulman and Y.-C. Chang, Phys. Rev. B 31, 2056 (1985).

${ }^{19}$ G. A. Sai-Halasz, L. Esaki, and W. A. Harrison, Phys. Rev. B 18, 2812 (1978).

${ }^{20}$ J. N. Schulman and T. C. McGill, Phys. Rev. B 19, 6341 (1979).

${ }^{21}$ K. C. Pandey and J. C. Phillips, Phys. Rev. B 13, 750 (1976).

${ }^{22}$ J. W. Harrison and J. R. Hauser, Phys. Rev. B 13, 5347 (1976).

${ }^{23}$ C. L. Anderson and C. R. Crowell, Phys. Rev. B 5, 2267 (1972).

${ }^{24}$ C. A. Lee, R. A. Logan, R. L. Bartdorf, J. J. Kleimack, and W. Wieggmann, Phys. Rev. 134, A761 (1964).

${ }^{25}$ A. R. Beattie, Semicond. Sci. Technol. 3, 48 (1988).

${ }^{26}$ R. Thoma, H. J. Peifer, and W. L. Engl, J. Appl. Phys. 69, 2300 (1991).

${ }^{27}$ V. Sankaran, J. M. Hinckley, and J. Singh, IEEE Trans. Electron Devices 40, 1589 (1993).

${ }^{28}$ J. M. Hinckley and J. Singh, Phys. Rev. B 41, 2912 (1990).

${ }^{29}$ J. M. Hinckley and J. Singh, Phys. Rev. B 42, 3546 (1990).

${ }^{30}$ J. M. Hinckley, Ph.D. thesis, University of Michigan, Ann Arbor, 1990.

${ }^{31}$ J. M. Hinckley and J. Singh, J. Appl. Phys. 76, 4192 (1994).

${ }^{32}$ L. V. Keldysh, Sov. Phys. JETP 10, 509 (1960).

${ }^{33}$ W. N. Grant, Solid-State Electron. 16, 1189 (1973).

${ }^{34}$ R. Van Overstraeten and H. De Man, Solid-State Electron. 13, 583 (1970).

${ }^{35}$ E. Cartier, M. Fischetti, E. Eklund, and F. McFeely, Appl. Phys. Lett. 62, 3339 (1993).

${ }^{36}$ S. L. Miller, Phys. Rev. 99, 1234 (1955).

${ }^{37}$ D. R. Decker and C. N. Dunn, IEEE Trans. Electron Devices 17, 290 (1970). 